\title{
DYNAMICAL PROCESSES RELATED TO CYCLONE DEVELOPMENT NEAR GREENLAND
}

\author{
Ragnhild Bieltvedt Skeie ${ }^{1}$, Jón Egill Kristjánsson ${ }^{1}$, Haraldur Ólafsson ${ }^{2,3}$ \\ ${ }^{1}$ Department of Geosciences, University of Oslo, P.O.Box 1022 Blindern, N-0315 Oslo, Norway. \\ ${ }^{2}$ Icelandic Meteorological Office, Bústadavegi 9, IS-150 Reykjavik, Iceland \\ ${ }^{3}$ University of Iceland and Institute for Meteorological Research, Reykjavik. \\ E-mail:r.b.skeie@geo.uio.no
}

\begin{abstract}
A cyclone that caused heavy snowfall and winds exceeding $30 \mathrm{~m} / \mathrm{s}$ over E-Greenland and N-Iceland on 2021 September 2003 is investigated. Numerical simulations are conducted to assess the role of Greenland's orography for the development, as well as to evaluate the significance of other factors such as latent heating, SST and SST gradients. The simulations reveal that the cyclone evolution is strongly affected by the orography of Greenland. When orography is removed, a deep, well organized baroclinic low develops rapidly and moves eastward at $75^{\circ} \mathrm{N}$. Conversely, in the control run the evolution of the primary baroclinic low is greatly suppressed by the orographic retardation of the warm air ahead of and the cold air behind the low. At the same time, a secondary low off Greenland's east coast at $68^{\circ} \mathrm{N}$ intensifies due to a coupling between an approaching upper level PV-anomaly and a lower level PV-anomaly generated from lee effects. This secondary low then moves eastward and causes extreme weather conditions, as observed. Further sensitivity experiments show that latent heating contributes to deepen the low, while SST gradients and SST in general contribute relatively little.
\end{abstract}

Keywords - Greenland, cyclone development, orography, latent heat

\section{INTRODUCTION}

An intense cyclone near the east coast of Greenland in September 2003 is investigated to determine atmospheric factors contributing to its development. The cyclone led to sustained winds exceeding $30 \mathrm{~m} / \mathrm{s}$ and heavy snowfall on the east coast of Greenland on the $20^{\text {th }}$ of September, while northern Iceland was hit by heavy snowfall and strong winds the following day. The purpose of this study is to investigate what role Greenland's orography and other factors may have played in the cyclone development. In this regard, comparisons will be made to recent investigations of the influence of Greenland on cyclone development through case studies (e.g., Kristjánsson and McInnes, 1999) and idealized studies (e.g., Petersen et al., 2003).

\section{MODEL}

The numerical model used in this study is the PSU/NCAR mesoscale model, MM5. The case is run with a $36 \mathrm{~km}$ horizontal grid resolution and $100 \times 100$ grid points in the horizontal, while in the vertical there are 23 sigma layers. The domain is centered at $68^{\circ} \mathrm{N}, 25^{\circ} \mathrm{W}$. The following physical parameterization schemes were used: Grell cumulus parameterization scheme, MRF PBL scheme, "simple ice" explicit moisture scheme and "cloud radiation" scheme (MM5 User's Guide 2003). The initial and boundary conditions are derived from the European Centre for Medium-Range Weather Forecast (ECMWF) reanalysis, and the lateral boundary conditions are updated every 6 hours. The model is run for 92 hours starting at 00 UTC 18 September.

\section{SIMULATIONS}

The case is run without mountains on Greenland (NOGREEN), without latent heat release (NOLAT), with reduced sea surface temperature (SST-RED) and with no SST gradients in the North-Atlantic (SST- 
GRAD) in addition to the control run with no changes (CONTROL). In the NOGREEN run the height of Greenland is set to $1 \mathrm{~m}$, and the model extrapolates the atmospheric conditions from ECMWF reanalysis down to sea level. In the NOLAT run, the latent heat release is excluded from the parameterization schemes. The RED-SST run is conducted with the initial SST reduced by $5 \mathrm{~K}$, and the SST-GRAD run is conducted with the mean SST in the east-west direction in the North-Atlantic used as initial conditions instead of the actual SST.

\section{RESULTS}
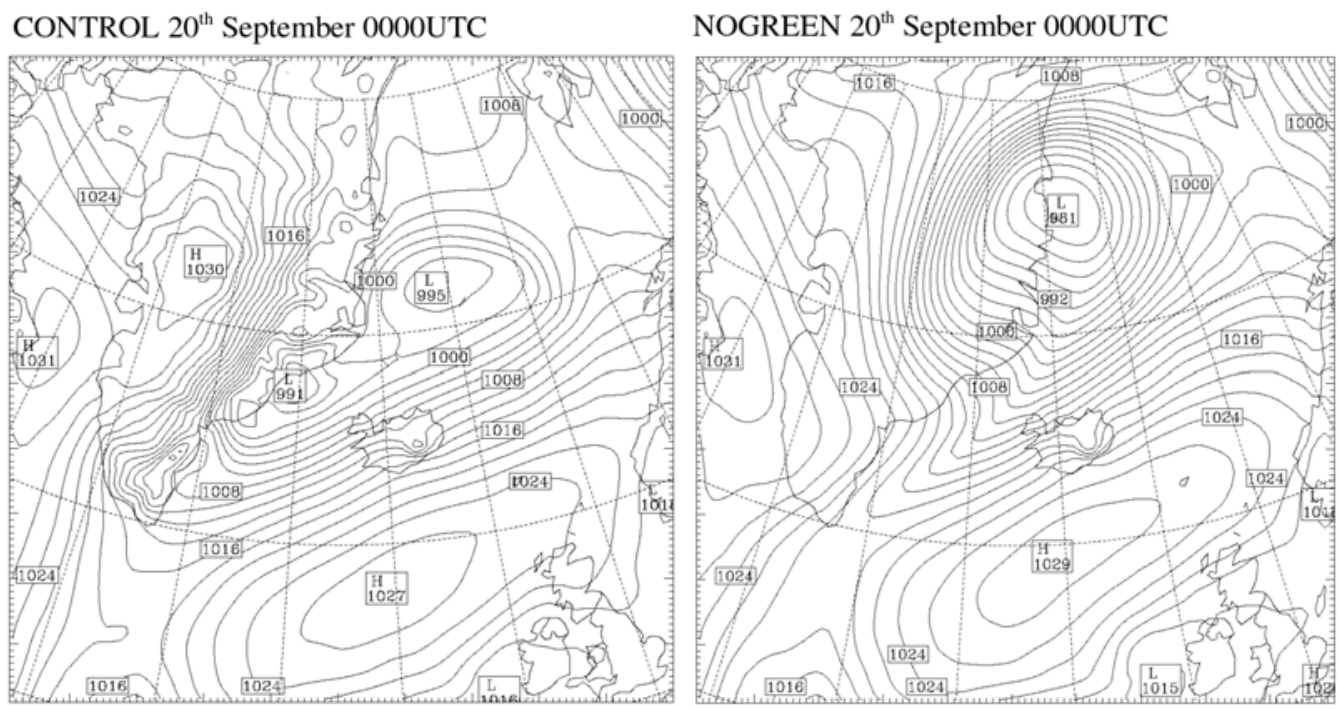

Figure 1. Sea-level pressure $[\mathrm{hPa}]$ in the control (left panel) and no-mountain (right panel) simulation at +48 hours.

In the control run, as in the analyses, a surface low passes over Greenland from the northwest corner of Greenland. When it reaches Greenland's east coast, a secondary low appears south of the primary baroclinic low. In Fig. 1 the sea level pressure at $20^{\text {th }}$ of September 00 UTC for CONTROL and NOGREEN is shown. Note that in CONTROL there are two lows on the east coast of Greenland, while in the NOGREEN run, the secondary low did not appear, indicating that it is a lee low, as in Petersen et al. (2003). Comparing the two panels of Figure 1, a dipole can be discerned along Greenland's east coast (not shown) between higher pressure to the north and lower pressure to the south in CONTROL, compared to NOGREEN. This is entirely analogous to the finding of Kristjánsson and McInnes (1999) in their case study. As they suggested, the orography reduces the cold advection behind the primary low, weakening the baroclinic energy conversion. Our analysis suggests that in this case also the warm air advection ahead of the low is similarly suppressed, while in the NOGREEN run this warm air contributes to the baroclinic growth of the cyclone. Consequently, the primary low weakens in CONTROL, but in contrast to Kristjánsson and McInnes (1999), the secondary low does not remain quasi-stationary. Instead, it intensifies rapidly as it starts to interact with an upper level trough approaching from the west. After 54 hours a well defined cold front has formed in connection with the secondary low, and at 61.6 hours the satellite picture (Figure 2) shows a well developed frontal system, while the primary low is now just a trough, located NE of Jan Mayen. The secondary low has now turned into a major developing baroclinic low. This is the low that caused the extreme weather conditions in E Greenland later in the day and over $\mathrm{N}$ Iceland the following day. The NOGREEN low reaches its mature stage 24 hours earlier than in the CONTROL run, but the surface low in the CONTROL simulation gets deeper than the NOGREEN low.

Figure 3 shows the development of the $500 \mathrm{hPa}$ height in the different model runs. Note how dramatically different the evolution in the NOGREEN run is, compared to all the other simulations. Between +24 and +48 hours there is a rapid deepening of the $500 \mathrm{hPa}$ low in the NOGREEN run, 
indicating a healthy baroclinic development, while in CONTROL, the low at $500 \mathrm{hPa}$ weakens as it crosses Greenland, and the primary low remains weak (Figure 1a). Once the interaction with the upper level trough spins up the secondary low, a rapid intensification takes place. This intensification is connected to a mutual interaction between a low level PV-anomaly related to mountain effects and an upper level PV-anomaly with air from the stratosphere. Figure 4 shows the PV-anomalies at 30 and 54 hours of the control-simulation. At 30 hours, at 06 UTC 19 September, there is a PV-anomaly at the surface in connection with the lee-low. The flow is blocked and the $\theta$-surfaces are collapsing, having a hydraulic jump-like structure, with PV generation (Epifanio and Durran, 2002). At upper levels the PVanomaly in connection with the polar jet has moved over Greenland. At 54 hours, at 00 UTC 21 September, the stability is low over Greenland's east coast and the upper level anomaly is able to penetrate down and spin up the circulation at all levels.

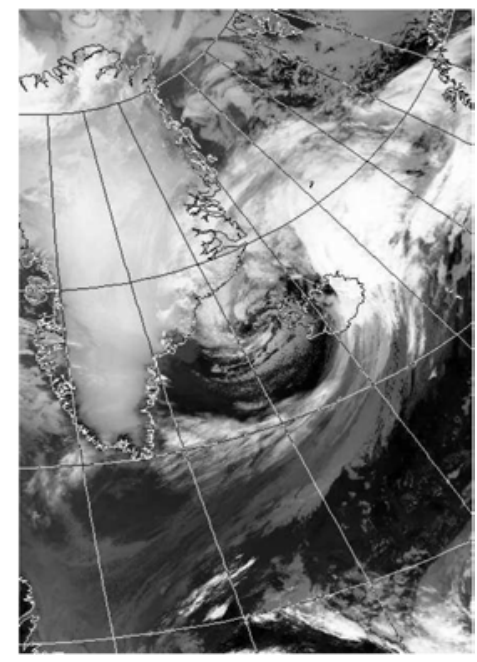

Figure 2. Infrared satellite picture at 13:39 UTC 20 September 2003 (Dundee Satellite Receiving Station).

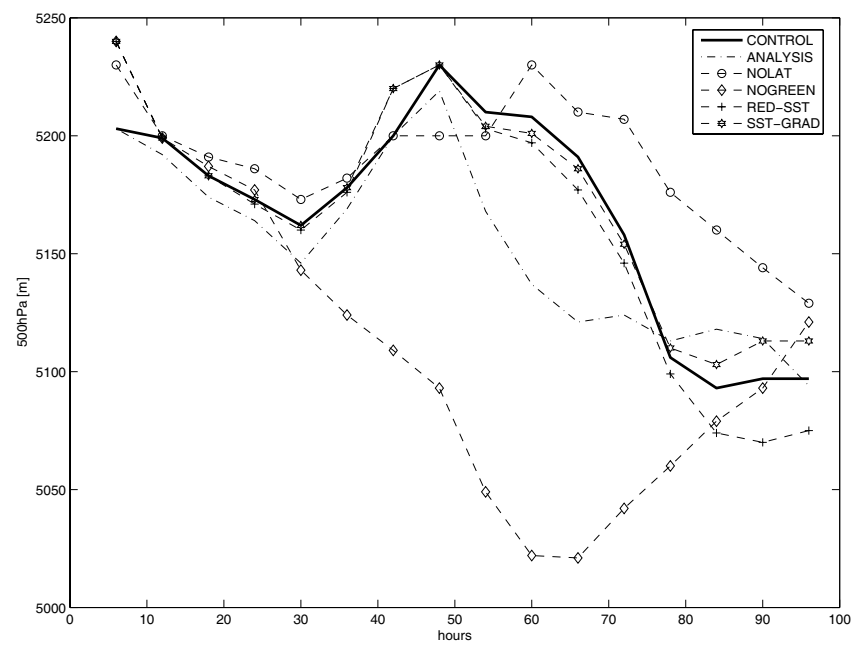

Figure 3. Geopotential height at $500 \mathrm{hPa}$ for the simulations and the analysis.

From theoretical considerations of PV redistribution one would expect the low to be intensified underneath the level of maximum latent heat release and weakened above this level. In the lower troposphere, excluding latent heating weakens the low, as expected. We find, however, a weakening of the low in NOLAT at all levels up to the tropopause (e.g., Figure 3). The reason for this is not clear, but it is conceivably related to the dominant role of orographic forcing in the cyclone development.

SST gradients and SST in general were found to contribute relatively little to cyclone deepening. The SST gradients were not very large in this September case, since the Arctic seawater is at its warmest at this time of the year. The SST gradients may play a more important role for cyclone developments in other parts of the year.

\section{CONCLUSION}

Simulations of the cyclone that led to a severe winter storm on 20-21 September 2003 in E-Greenland and N-Iceland have revealed the following: In the aftermath of a baroclinic development that is greatly suppressed by Greenland's orography, a secondary lee-cyclone is formed between Iceland and Greenland. The secondary cyclone deepens explosively as it interacts with an upper-level PV anomaly that approaches from the west. The severe weather occurs in connection with the back-bent warm front at the rear of the cyclone as it moves ENE. 
a)

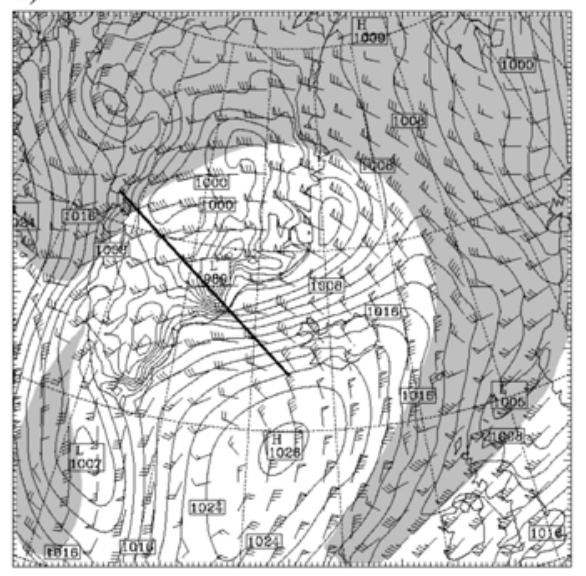

c)

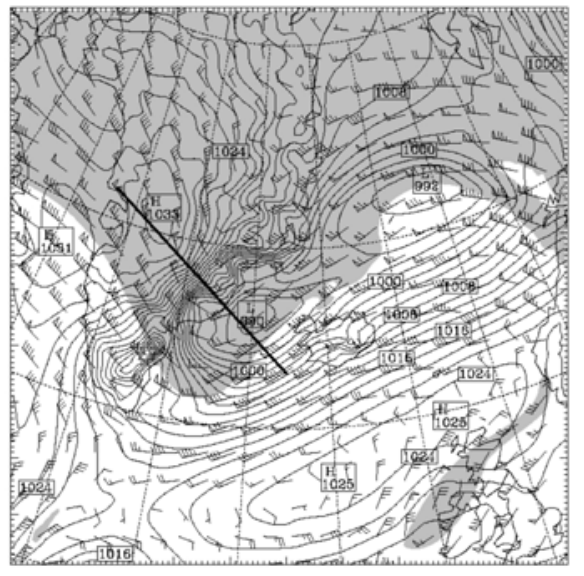

b)

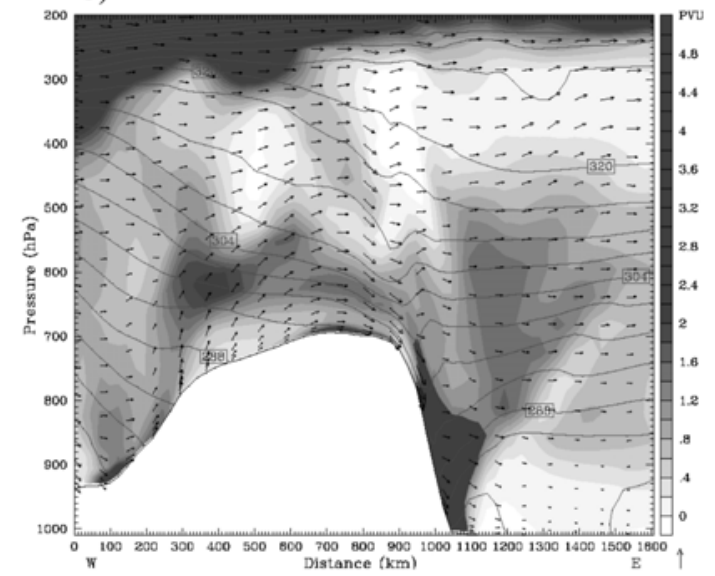

d)

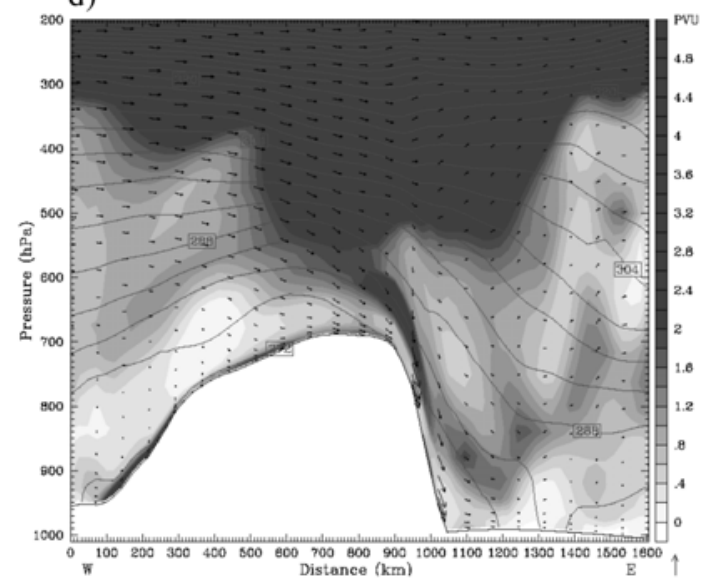

Figure 4. Left column: potential vorticity $>2$ PVU (shading) at $\theta=316 \mathrm{~K}$, horizontal wind at at $\theta=316$ $\mathrm{K}$ (full barb $=5 \mathrm{~m} / \mathrm{s}$ ) and sea-level pressure. Right column: cross sections, marked in a) and c), potential vorticity (shading), potential temperature $[\mathrm{K}]$ and wind vectors. Valid time: a) and b) 06 UTC 19 September ( +30 hours simulation time); c) and d) 06 UTC 20 September ( +54 hours simulation time).

\section{REFERENCES}

Epifanio, C. C., and D. R. Durran, 2002: Lee-vortex formation in free-slip stratified flow over ridges. Part II: Mechanisms of vorticity and PV production in nonlinear viscous wakes. J. Atmos. Sci., 59, $1166-1181$.

Kristjánsson, J. E., and H. Mc Innes, 1999: The impact of Greenland on cyclone evolution in the North Atlantic, Quart. J. Roy. Meteorol. Soc., 125, 2819-2834.

MM5 user's guide 2003: PSU/NCAR Mesoscale Modeling System, Tutorial Class Notes and User's Guide: MM5 Modeling System Version 3.

Petersen, G. N., H. Ólafsson, and J. E. Kristjánsson, 2003: Flow in the lee of idealized mountains and Greenland. J. Atmos. Sci., 60 (No. 17), 2183-2195. 